\title{
A Review on Artificial Bee Colony Algorithms and Their Applications to Data Clustering
}

\author{
Ajit Kumar ${ }^{1}$, Dharmender Kumar ${ }^{2}$, S. K. Jarial ${ }^{1}$ \\ ${ }^{1}$ Deenbandhu Chhotu Ram University of Science and Technology, Murthal, India \\ ${ }^{2}$ Guru Jambheshwar University of Science and Technology, Hisar, India \\ Emails: ajit.hisar@gmail.com dharmindia24@gmail.coms.jarial@rediffmail.com
}

\begin{abstract}
Data clustering is an important data mining technique being widely used in numerous applications. It is a method of creating groups (clusters) of objects, in such a way that objects in one cluster are very similar and objects in different clusters are quite distinct, i.e. intra-cluster distance is minimized and inter-cluster distance is maximized. However, the popular conventional clustering algorithms have shortcomings such as dependency on center initialization, slow convergence rate, local optima trap, etc. Artificial Bee Colony $(A B C)$ algorithm is one of the popular swarm based algorithm inspired by intelligent foraging behaviour of honeybees that helps to minimize these shortcomings. In the past, many swarm intelligence based techniques for clustering were introduced and proved their performance. This paper provides a literature survey on $A B C$, its variants and its applications in data clustering.
\end{abstract}

Keywords: Data clustering, swarm intelligence, artificial bee colony, metaheuristics, optimization.

\section{Introduction}

Swarm Intelligence (SI) is an emerging research field inspired by collective intelligence of social insects or animals in group, such as ant colonies, bee colonies, bird flocks, fish schools and so on. The concept of collective intelligence was originally introduced in [1] to discuss the self-organizing and intelligent behavior of ants and explain how the ants choose between different routes to food sources with varying quality. However, the concept was gradually discarded and it started reemerging in the late 1980s with the increase in research on artificial life and complex systems theory. Collective intelligence may be considered as the collection of large number of agents that interact among themselves, as well as active environment to generate adaptive behavior [2]. The term "swarm intelligence" was introduced in the context of cellular robotic systems [3]. The relation between an individual and the society was better explained in terms of collective exploration 
and group behavior of social insects. Their incredible characteristics such as selforganization, collective decisions, division of labor, positive and negative feedback play a major role in designing of complex systems for numerous applications [4]. A modern concept of collective intelligence was provided in terms of swarm as " a swarm is a set of (mobile) agents which are liable to communicate directly or indirectly (by acting on their local environment) with each other, and which collectively carry out a distributed problem solving" [5]. In addition, they describe Swarm Intelligence as "a property of non-intelligent agents exhibiting collectively intelligent behavior".

Swarm Intelligence is also defined as "any attempt to design algorithms or distributed problem-solving devices inspired by the collective behavior of social insect colonies and other animal societies" [6]. H in c h e y, S t e r r it t and R o u f f [7] have defined swarm as "a large group of simple components working together to achieve a goal and produce significant results". Some authors in [8] have described swarm intelligence in the context of both humans and animals as "two or more individuals independently, or at least partially independently, acquire information and these different packages of information are combined and processed through social interaction, which provides a solution to a cognitive problem in a way that cannot be implemented by isolated individuals". Most widely used SI algorithms developed during recent years are: Ant Colony Optimization, which is based on ant colonies [9], Particle Swarm Optimization, which is based on fish schooling/bird flocking [10], Immune Algorithm, which is based on swarm of cells and molecules [11], Bacterial Foraging Optimization, which is based on E. Coli bacteria foraging (see [12]), Artificial Bee Colony, which is based on honey bee swarms [13], Cat Swarm Optimization, which is based on behavior of cats [14], Cuckoo Search Algorithm [15], Firefly Algorithm, which is based on flashing behavior of tropical fireflies [16], Gravitational Search Algorithm which is based on interaction of masses [17] and many more.

In this digital era, it is really challenging to store, process and analyze the huge and rapidly increasing data without appropriate tools or techniques. Data mining is the process of extracting useful information and knowledge by exploring and analyzing this data using analytical algorithms or means. Clustering or cluster analysis is a form of indirect data mining, as the goal is to find the relationships among all the variables in contrast to direct data mining, where some variables are pointed out as targets [18]. Clustering is an important and popular data mining task. It can be regarded as a form of unsupervised classification i.e. labeling of objects does not rely on predefined classes, rather it derives from the data itself $[19,20]$. Clustering is a method of creating groups (clusters) of objects, in such a way that objects in one cluster are very similar (or related) and objects in different clusters are quite distinct (or unrelated). This process mainly consists of four steps. In the first step, distinguishing features are selected from a set of candidates and then extracted using some transformations. The second step is to select a proximity measure and construction of a criterion function. Third step is defining testing criteria for cluster validation and finally, the results are interpreted to check reliability of useful information [21]. Clustering is widely used in numerous 
applications, including market research, pattern recognition, data analysis, document retrieval, image segmentation, artificial intelligence, bioinformatics, classification of coals, financial investment, data compression, web mining, machine learning and image processing [22-24].

Objects similar to each other are identified in a cluster or group of a data set (different from those in other clusters/groups) using clustering techniques. Euclidean distance is the most used similarity metric derived from the Minkowski metric; the lesser the distance, more similarity is there between two objects or two clusters. Clustering is categorized as hard clustering and fuzzy clustering. Hard clustering is sub-categorized as hierarchical clustering and partitional clustering $[18,23]$. In hierarchical clustering objects are gradually (dis)assembled into clusters whereas objects are iteratively relocated to form clusters of proper convex shapes in partitional clustering. Hierarchical clustering can be divided into agglomerative methods and divisive methods. Agglomerative (bottom-up) clustering begins with each object as a separate cluster and starts merging the most similar clusters recursively according to some criteria [25]. Divisive (top-down) clustering begins with the whole data set in one cluster and splits it into successively smaller clusters. Clearly, this method is less frequently used than agglomerative method since poor choices at early stages may not provide optimal solutions [26]. However, there may be drawbacks in both methods: incorrect grouping of data points at early stage that may not be reallocated, difficulty of choosing right stopping criteria, different measures for similarity between clusters generating different results [18, 27, 28].

Partitional algorithms divide the set of data in clusters by iteratively relocating objects without hierarchy. The clusters are gradually improved to ensure high quality of clustering. The clusters are formed in such a way that each cluster contains at least one data object and each data object belongs to exactly one cluster [29]. F i s h e r [30] expressed the set of data elements into groups such that squared distance (sum of squares) within each group is minimized. The one-dimensional approach involved in the process may be used to solve $\mathrm{N}$-dimensional grouping. An early version of k-Means was proposed by Forg y [31] called as two step method of clustering. The method reassigns the data points to their nearest cluster centres in first step, while the centres of the newly formed groups are recomputed in the next stage. The process continues until convergence criterion is met. Macque en [32] defined the k-Means to generate partitions of an $\mathrm{N}$-dimensional population such that each partition is having small within-class variance. The centres of so formed partitions are continuously updated with the assignment of data points to different partitions. In k-Means, each cluster has a centre called mean and attempt is made to minimize its objective function (a square error function). The objective function for a set of data objects $X=\left\{x_{1}, \ldots, x_{N}\right\}, K$ disjoint subsets $c_{j}$ is given as

$$
K M(X, C)=\sum_{i=1}^{N} \min \left\{\left\|x_{i}-c_{j}\right\|^{2} \mid j=1, \ldots, K\right\} .
$$

The k-Means algorithm also has some limitations: dependence on initialization of cluster centers, sensitivity to outliers, non-guaranteed optimal solutions, formation of unbalanced clusters [22, 28, 33]. There have been several approaches to overcome the above shortcomings, which are based on swarm, insects and 
natural phenomena. A Genetic k-Means algorithm was proposed in [34] that makes use of a search operator and a biased mutation operator to find global optimal solutions in clustering. Garai and Chaudhuri [35] introduced a two-phase genetically based clustering algorithm. The data is first decomposed into a large number of clusters using Cluster Decomposition Algorithm and then combined using Hierarchical Cluster Merging Algorithm. During the process, genetic algorithm is applied on fragmented clusters in several cycles to find the final partitions. One GA-clustering algorithm integrating the capability of GA with the simplicity of k-Means was developed to avoid the sub optimal solutions in clustering [36]. A genetic algorithm exchanging neighboring centers for k-Means clustering was proposed in [37]. Selim and Alsultan [38] introduced a simulated annealing algorithm for data clustering. A number of algorithms based on tabu search method have been proposed for data clustering [39-41]. Some approaches to calculate initial centers for k-Means clustering exist in the literature [42, 43]. Zalik [44] introduced an extension of k-Means to perform clustering without pre-assigning the correct number of clusters. Some authors in [45] proposed an ACO based algorithm for data clustering and found encouraging results. A large number of approaches based on PSO algorithm have been developed for data clustering [46-50].

Although the above approaches solve the problems of k-Means up to some extent but they also encounter some drawbacks. Genetic algorithms suffer from expensive evolutionary operators and costly fitness function. Simulated annealing suffers from slow convergence speed and non-availability of computation function for stopping criteria. Limitations of tabu search include requirement of external memory and slowness in finding optimal solutions. ACO suffers from possibility of falling in local optimal solutions and tendency to stagnancy. Some of the limitations in PSO are premature convergence on early suboptimal solutions and poor results in complex data set [51-53].

In the past, several new intelligent approaches simulating the behaviour of swarm systems have been proposed. Artificial Bee Colony is a population-based algorithm introduced by Karaboga [13], which is inspired by the intelligent foraging behaviour of honeybees. $\mathrm{ABC}$ has been successfully used in wide applications such as neural networks, sensor networks, protein structure, image processing, data mining, industrial engineering, mechanical engineering, civil engineering and electrical engineering [54-57]. Solution to complex transportation problems as well as deterministic combinatorial problems in dynamic environments is made possible by intelligent features of bee swarm such as autonomy, self organizing, distributed functioning, division of labour, etc., [58-60].

There have been several surveys of $A B C$ available in the literature. $\mathrm{K}$ a r a b og a et al. [61] have presented a comprehensive survey of $\mathrm{ABC}$ in terms of modifications, hybrid models and applications in various fields. In [62] the survey has given general features of $\mathrm{ABC}$ and comparative analysis of its application in different areas. The survey in [63] highlights the importance of $A B C$, its hybrid approaches and applications in wide areas. Kumar and $\mathrm{Kumar}$ [64] have presented modifications of $\mathrm{ABC}$ to solve a variety of problems and applications in 
selected areas. However, the present survey provides the comprehensive insight about the variants of $\mathrm{ABC}$ as well as its applications in solving data clustering problems.

\section{Artificial bee colony algorithm}

Honeybees perform a number of complex tasks in systematic manner, best example is collection of nectar and its processing [65]. The information about quality of food sources is shared among its colony members by means of dance language that acts as a communication behaviour [66]. The foraging range with proper precision allows a colony to exploit potential food sources with great efficiency and helps to concentrate its foraging on best patches [67]. Food transmission is the most effective way of communication in honeybee colonies and determines the requirements of the colony. Such requirements and the age of individual bees determine the division of labour in honeybees and thus their social life [68]. Study in [69] proves that the colony acts as a unit and among its members so that there is mutual dependence on each other. Their behaviour is closely related to the needs of the colony. A mathematical model developed in [70] enables a forager to adopt cooperative or direct recruitment of food sources. The effectiveness and simplicity of the whole process is possible because of the decentralized decision-making approach [71] and systematic mechanism of self-organization in honeybee colonies [72]. The honeybee swarm has a specific physical structure and not just a random collection of individuals [73].

Self-organization, a key feature of swarm system represents the emergence of complex collective behaviour from local interactions among agents exhibiting simple behaviours [74]. More interesting is that the resulting behaviour emerges without any control hierarchy, but with the autonomous functioning of individuals [72]. Another important feature of swarm is division of labour, a process in which specialized agents perform simultaneous activities resulting in performance that is more efficient as well as saving of time $[6,75]$. The ratios of individual agents performing various activities are adjusted in order to respond to internal and external changes. This behavioural flexibility of individual agents is termed as plasticity, a key aspect of division of labour [76].

$\mathrm{ABC}$ is one of the widely studied algorithms, which is continuously inspiring researchers to apply in solving several real world problems. There are three groups of artificial bees in ABC: employed bees, onlooker bees and scout bees. Bees going to a food source already visited by them are employed while the bees looking for a food source are unemployed. Scout bees carry out search for new food sources, onlooker bees wait for information about food sources given by the employed bees through waggle dance. It means that number of employed bees are same as number of food sources. An employed bee becomes scout when the position of a food source does not improve through the predetermined number of attempts called "limit". In this way, exploitation process is performed by employed and onlooker bees whereas scouts perform exploration of solutions. The details of $\mathrm{ABC}$ algorithm are given as under: 
- Initialization phase

The locations of food sources are randomly initialized within the range of boundaries according to the equation

$$
x_{i j}=x_{j}^{\min }+\operatorname{rand}(0,1)\left(x_{j}^{\max }-x_{j}^{\min }\right),
$$

where $i=1, \ldots, \mathrm{SN}$ and $j=1, \ldots, D$. SN indicates the number of food sources and taken as half of the bee colony, $D$ is dimension of the problem, $x_{i j}$ represents the parameter for $i$-th employed bee on $j$-th dimension, $x_{j}^{\max }$ and $x_{j}^{\min }$ are upper and lower bounds of $x_{i j}$.

- Employed bee phase

Each employee bee is assigned to the food source for further exploitation. The resulting food source is generated according to the equation

$$
v_{i j}=x_{i j}+\varphi\left(x_{i j}-x_{k j}\right),
$$

where: $k$ is a neighbour of $i, i \neq k ; \varphi$ is a random number in the range $[-1,1]$ to control the production of neighbour solutions around $x_{i j}$; $v_{i j}$ is the new solution for $x_{i j}$. The fitness of new food source is now calculated using the equation

$$
\mathrm{fit}_{i}=\left\{\begin{array}{cc}
\frac{1}{1+f_{i}}, & f_{i} \geq 0, \\
1+\operatorname{abs}\left(f_{i}\right), & f_{i}<0,
\end{array}\right.
$$

where $f_{i}$ is the objection function associated with each food source, and fit is the fitness value. A greedy selection is performed on $x_{i j}$ and $v_{i j}$, i.e., original and new food sources to choose better one according to its fitness value.

- Probabilistic selection phase

For each food source, a probability value is calculated using the next equation, and an onlooker bee selects the food source according to this value:

$$
p_{i}=\frac{\mathrm{fit}_{i}}{\sum_{j=1}^{N} \mathrm{fit}_{j}},
$$

where fit $i_{i}$ is the fitness value of $i$-th solution and $p_{i}$ is the selection probability of $i$-th solution.

- Onlooker bee phase

The employed bees share the information about food sources with the onlooker bees for further processing. Each onlooker bee selects a food source to exploit according to the probability associated with it (i.e., more fitness, higher the probability). The chosen food sources are exploited for better solutions using Equation (3) and their fitness values are calculated using Equation (4). A greedy selection is again applied on the original as well as new food sources, similar to employed bee phase.

- Scout bee phase

If a food source does not produce better solutions even up to a predefined limit, the food source is abandoned and the corresponding bee becomes a scout bee. A new food source is randomly generated in the search space using Equation (2). 
The employed, onlooker, scout bee phases and probabilistic selection phase will execute until termination criterion is satisfied. The best food source solution is obtained as output.

\section{3. $\mathrm{ABC}$ variants}

$\mathrm{K}$ a r a b og a [13] invented $\mathrm{ABC}$ algorithm based on intelligent foraging behaviour of honeybee swarm and the same was applied for solving unconstrained optimization problems in [77]. The authors in [78] tested the algorithm for multivariable function optimization and comparison was made with some popular methods such as GA, PSO and PS-EA. The results prove the better performance of $\mathrm{ABC}$ in comparison to other approaches. Karaboga and Basturk [79] successfully applied $\mathrm{ABC}$ in solving a set of constrained optimization problems. $\mathrm{Karaboga}$, Akay and Ozturk [80] successfully applied the ABC algorithm to train neural networks and compared its performance with the genetic algorithm and back propagation algorithm. Karaboga and Basturk [81] compared the performance of $\mathrm{ABC}$ with algorithm like DE, PSO and EA and concluded that $\mathrm{ABC}$ is efficient to solve multimodal and multi-dimensional numeric problems. The study also pointed the colony size of 50-100 to provide reasonable convergence speed and moderate value of limit $\mathrm{L}$ for the scouts. A study on performance of $\mathrm{ABC}$ in solving multimodal and multidimensional optimization problems was made in [82] and compared with DE, ES, GA and PSO algorithms on a set of test functions. Since its inception, several variants of $\mathrm{ABC}$ have been developed to overcome its shortcomings and improve the performance. Some of these variants contain modifications of the original $\mathrm{ABC}$ while others contain hybridization of $\mathrm{ABC}$ with traditional and evolutionary algorithms. An Improved $\mathrm{ABC}$ algorithm proposed by $\mathrm{Liu}$ et al. [83] employed chaotic mapping having sufficient population randomness and improved ergodicity during initialization. The improved search equation in scout bee phase provided better convergence. One of the variants, gbest-guided $\mathrm{ABC}(\mathrm{GABC})$ algorithm improves the exploitation of $\mathrm{ABC}$ by applying global Best solution (gBest) inspired by PSO. The gbest term introduced in the solution search equation of $\mathrm{ABC}$ enhances the exploitation while maintaining the balance between exploration and exploitation of solution space. The algorithm was found superior on most benchmark functions [84]. The algorithm was further modified by adopting the information of both global best and best between current and random bee solution [85]. Taking inspiration from the global best ABC algorithms, E1-A bd [86] modified $\mathrm{ABC}$ in grouping of bees into different sub-populations and use of local best information for update of search equations.

Fister et al. [87] introduced new crossover operator and mutation techniques in $\mathrm{ABC}$ to enhance performance. They also proposed to hybridize $\mathrm{ABC}$ with Nelder-Mead algorithm and RWDE method to enhance exploration and exploitation capabilities. B ansal et al. [88] proposed a new version of $\mathrm{ABC}$ namely Memetic $\mathrm{ABC}$ by adding an additional search phase to improve the exploitation process. The proposed phase makes use of one specific method called GSS approach to exploit the solution space in the neighbourhood of best solutions. 
Kumar, Sharma and Kumari [89] introduced two new parameters in the memetic search phase to improve the local search process. The proposed RMABC algorithm makes use of Golden Section Search method and new parameters to search best solutions. Kojima, Nakano and Miyauchi [90] modified ABC by eliminating the use of scout bee phase and improving employed bee as well as onlooker bee phases in such a way to maintain balance between solution search speed and speed of convergence. The algorithm was successfully tested in dynamic optimization problems. Y u, Z h a n g and $\mathrm{Ch}$ e $\mathrm{n}$ [91] introduced a new position update strategy for onlooker bees to improve the exploitation process and enhance the convergence. The technique incorporates the good solutions as opposed to random solutions in position update of onlooker bees and at the same time adjusting the greediness degree using a control scheme.

In order to solve the real world constrained optimization problems, Brajevic and Tuba [92] developed a new UABC algorithm by enhancing the exploitation in onlooker bees and exploration in scout bees to maintain the balance. The algorithm proved its effective and robust performance in solving constrained engineering problems. Karaboga and A kay [93] modified ABC algorithm by incorporating Deb's heuristic rules and probability selection scheme for constrained optimization problems. Li and $\mathrm{Y}$ in [94] modified ABC for constrained optimization problems in such a way that employed bees generate solutions based on feasible rule method, whereas onlooker bees perform search in accordance with multi-objective optimization method. A kay and Karaboga [95] modified the $\mathrm{ABC}$ algorithm by incorporating Deb's three heuristic rules to make the feasible search. The resulting improved exploration and exploitation capability of $\mathrm{ABC}$ algorithm prove better performance as compared to DE and PSO algorithms on large scale unconstrained optimization problems.

Some researchers also modified $\mathrm{ABC}$ algorithm to solve binary optimization problems. Kashan, Nahavandi and Kashan [96] introduced DisABC, a modified version of $\mathrm{ABC}$ for binary optimization problems. The algorithm makes use of a differential expression employing dissimilarity measure between binary structures and successfully applied on UFLP problem. Pampara and Engelbrecht [97] presented three versions of $A B C$ for binary-valued optimization problems and found the performance of angle modulated $\mathrm{ABC}$ better than normalized and binary $\mathrm{ABC}$. Ch andras e karan et al. [98] developed two variants of $\mathrm{ABC}$ namely binary coded $\mathrm{ABC}$ and real coded $\mathrm{ABC}$ to solve the unit commitment and economic dispatch problem. Kim et al. [99] proposed binary $\mathrm{ABC}$ algorithm for job scheduling problems. They introduced efficient BABC by use of flexible ranking strategy for selection of jobs in order to maintain exploration and exploitation of the search solutions.

Singh [100] successfully implemented ABC algorithm for the leafconstrained minimum spanning tree problem, which is a discrete optimization problem. P a n et al. [101] proposed a discrete version of ABC to solve flow shop scheduling problems. The problems were solved by utilising the effective population initialization approach and self-adaptive neighbouring food source generation strategy of ABC. Yurtkuran and Emel [102] modified ABC for 
use in solving a combinatorial problem such as p-center problem. In this process, solutions are represented using random key-based encoding and different search techniques are employed in search process to generate new solutions. $\mathrm{Li}, \mathrm{Pan}$ and Gao [103] developed a Pareto-based discrete ABC (P-DABC) algorithm by combining the discrete version of $\mathrm{ABC}$ and an external pare to archive set to solve multi-objective flexible job shop scheduling problems. In the proposed algorithm, the food source is represented by two components: routing component and scheduling component. One crossover operator is employed for effective sharing of information in routing component.

B eloufa and Chikh [104] modified ABC to enhance the exploration and exploitation processes of employed or onlooker bees by employing a blended crossover operator. The modified algorithm was used to create an effective fuzzy classifier and successfully applied in diagnosis of diabetes disease. K h or s andi, Hosseinian and Ghazanfari [105] modified the search equation in original $\mathrm{ABC}$ to improve the exploration and exploitation process while solving optimal power flow problem using fuzzy based method.

$\mathrm{Di}$ old et al. [106] proposed two variants called $\mathrm{ABC}_{\mathrm{gBest}}$ and $\mathrm{ABC}$ gBestDist to study the selection of reference locations that may affect the position of artificial bees. They also studied the influence of population size, ratio of employed and onlooker bees in $\mathrm{ABC}$ optimization. The variants make use of global best reference and distance based reference to generate new solutions. Another variant, Hybrid Differential ABC (HDABC) presents combination of ABCA with Differential Evolution strategy. The algorithm was tested on various benchmark functions and proved efficiency in terms of better convergence and quality of solutions [107]. Some authors in [108] proposed Enhanced ABC (EABC) algorithm incorporating two modifications to enhance the neighbourhood searching as well as new-foodsource inducting performance of $\mathrm{ABC}$. The algorithm was tested and provided better performance. An enhanced global-best $\mathrm{ABC}$ optimization algorithm (EBABC) invented by Abro and Mohamad-Saleh [109] takes into consideration self-reinforcement, positive and negative feedback through mutation equations. EBABC was compared with several algorithms and proved better performance.

Li, Niu and Xiao [110] proposed a hybrid algorithm called PS-ABC algorithm that is having the concept of prediction and selection. The algorithm incorporates the features of three variants of $\mathrm{ABC}$, i.e., basic $\mathrm{ABC}, \mathrm{GABC}$ and $\mathrm{I}-\mathrm{ABC}$ to solve optimization problems. I-ABC algorithm improves the exploitation capability of basic $\mathrm{ABC}$ by introducing inertia weight and acceleration coefficients in the search process. PS-ABC makes use of three solution search equations to improve the search process as well as convergence speed and generate the best solution. The shortcomings of PS-ABC algorithm such as premature convergence and slower convergence were removed in Enhanced Probability Selection Artificial Bee Colony algorithm (EPS-ABC) developed by Abro and Moh a mad-S a leh [111]. The algorithm employs the modified equations to enhance exploration and avoid local optima traps. The intelligent scout bee stage of PS-ABC helps to find the optimal solutions. Main idea introduced in the algorithm is to generate solutions 
around random picked food source and gbest food source in addition to the current food source. S h ar m a, B a n s l and A r y a [112] proposed three modifications in the basic $\mathrm{ABC}$ by incorporating levy flight local search strategy, opposition based learning method and a position update equation to guide the search process. The local search method improves the exploitation capability by recalculating the step size while opposition based learning enhances the convergence speed. The performance of $\mathrm{ABC}$ was improved by use of NABC algorithm proposed by $\mathrm{Xu}$, $\mathrm{F}$ a $\mathrm{n}$ and $\mathrm{Yuan}$ [113]. NABC employed the hybridization of DE/best/1 scheme and $\mathrm{ABC}$ with a solution pool having best solutions. The solution pool provided the scope for the bees to search the neighbourhood of different best solutions and not just global best solution. $\mathrm{Kang}, \mathrm{Li}$ and $\mathrm{Li}$ [114] proposed a memetic algorithm by combining $\mathrm{ABC}$ with Hooke-Jeeves pattern search method for global optimization problems. The algorithm employed $\mathrm{ABC}$ to explore regions of attraction and pattern search method to exploit the regions for best solution.

Tsai et al. [115] proposed Interactive $\mathrm{ABC}$ algorithm and improved the exploration capability of $\mathrm{ABC}$ algorithm by applying the law of universal gravitation in onlooker bees. A lat a s [116] improved the convergence speed and avoided local optima traps in $\mathrm{ABC}$ by using sequences from chaotic number generators in place of random numbers. Chaotic sequences are deterministic in nature and possess spread-spectrum characteristics. Kiran and Gunduz [117] modified $\mathrm{ABC}$ algorithm by inserting crossover operation on certain employed bees with good solutions to generate neighbour for onlooker bees. The modified algorithm namely CABC was applied to solve energy demand problem and provided better convergence. Dongli et al. [118] proposed two modified ABC algorithms to improve the global exploration capability and convergence speed. In first algorithm, search range is made wider for improved exploration of employed bees while in second, sensibility replaced random number in searching of onlooker bees. In order to widen the search space to generate new solutions, some authors in D o ngli et al. [119] introduced the concept of multi-exchange neighbourhood resulting in improved global search and better convergence. B anharnsakun, A chalakul and Sirinaovakul [120] proposed a best-so-far ABC algorithm by introducing three major changes such as best-so-far method for the onlooker bees, adjustable search radius for scout bees and objective-value-based comparison of new solution with old solution to generate better solutions with fast convergence.

One improved version of $\mathrm{ABC}$ was proposed by $\mathrm{Gao}$ and $\mathrm{Liu}$ [121] to provide stability, accuracy and robustness. The IABC algorithm employed new initialization approach based on chaotic systems and opposition-based learning method. The algorithm also employed two new solution search equations for better convergence and population diversity. The algorithm was modified by presenting a new algorithm namely $\mathrm{ABC} /$ best in which two solution search equations were replaced in order to improve the exploitation process [122]. The focus of algorithm was on the search around best solution of the previous iteration. Gao and $\mathrm{Liu}$ [123] developed MABC by employing an initialization approach based on chaotic systems and opposition learning methods for better convergence. In addition, a modified search equation is also induced to enhance exploitation capability. To 
overcome the issue of poor exploitation in $\mathrm{ABC}, \mathrm{Gao}, \mathrm{Liu}$ and $\mathrm{Huang}$ [124] introduced a modified search equation to develop CABC. The convergence was enhanced by employing orthogonal learning strategy in CABC. Sharma and P a n t [125] developed I-ABC and I-ABC greedy algorithms to find the optimal solutions using $\mathrm{ABC}$, where the initial food sources are generated by use of opposition based learning method. I-ABC greedy makes use of greedy bee concept to find the best solution and enhanced convergence. Xiang and An [126] added four modifications to the basic $\mathrm{ABC}$ in order to enhance convergence performance, population diversity, exploration capability and avoiding local minima traps. The modifications such as chaotic initialization, roulette wheel based reverse selection, modified search equation and chaotic search make the ERABC as efficient and robust optimization algorithm.

B ansal et al. [127] introduced a new control parameter namely cognitive learning factor and modified the range of existing control parameter in $\mathrm{ABC}$ to propose $\mathrm{BABC}$ algorithm. The above modifications help to maintain the balance between exploration and exploitation capabilities of ABC. B is was et al. [128] proposed a modified algorithm MiMSABC to ensure effective exploration and exploitation in multi-swarm populations. Initially groups of foragers having their perturbation schemes were taken. In order to enhance the performance and better exchange of information, rank based migration scheme was used in the algorithm. $\mathrm{Lu} \mathrm{o}, \mathrm{W}$ ang and $\mathrm{Xiao}$ [129] proposed a new algorithm COABC by introducing a new search equation to improve the exploitation process in onlooker stage. The update process in onlooker phase employed the global best solution to find new candidate solutions. Sulaiman, Saleh and Abro [130] proposed a new version of $\mathrm{ABC}$ to enhance the fitness of poor solutions by using a modified mutation scheme. This way, the fitness of every possible solution is increased and provided faster convergence and avoidance of premature convergence. Ga o, Li u and $\mathrm{Hu}$ ang [131] introduced a new search equation in onlooker bees phase of $\mathrm{ABC}$ to improve the search process. A local search algorithm called Powell's method is also incorporated in the hybrid algorithm to improve the exploitation ability. Das and Chaudhur [132] presented hybridization of modified Quadratic Approximation with slight change in scout bees activities of $\mathrm{ABC}$ and generated better results.

Akay and Karaboga [133] proposed a modified ABC algorithm that studied the effect of various parameters such as perturbation rate, limit and scaling factor while solving real parameter optimization problems. In addition to the single parameter called limit being used in basic $\mathrm{ABC}$ algorithm, the modified algorithm introduced an additional parameter (i.e., modification rate) to control the frequency of perturbation. Another parameter to control the magnitude of perturbation is scaling factor. The modified algorithm performed better on hybrid functions as compared to PSO, DE and ES algorithms. Alizadegan, Asady and A hmadpour [134] proposed two modified ABC algorithms. In the first one, they used different ratios of employed and onlooker bees. The results prove the better performance of $\mathrm{ABC}$ having more onlooker bees. The second algorithm employed the changes of dimension's value during each iteration and generated 
positive results. Liang, Liu and Zhang [135] introduced the dynamic grouping strategy using cooperative coevolving to solve large-scale optimization problems. The main concept to generate better candidate solutions was mutual learning as well as global best positioning. Aydin et al. [136] developed a new version of $A B C$ called IABC-LS to enhance the intensification ability and better convergence. The local search procedure in the algorithm helped to find better solutions and provided better performance.

Since ABC is efficient in solving single objective problems, it has also been successfully applied in multi-objective optimization problems. This was made possible due to many characteristics of $\mathrm{ABC}$ such as use of less control parameters, absence of gradient-based information and well balancing of exploration and exploitation capabilities. Vector Evaluated Artificial Bee Colony (VEABC) algorithm for multi-objective optimization is based on concept of VEGA and VEPSO algorithms. The optimal solution is generated as the communication of information takes place between separate swarms employed for multiple objectives [137]. Hed a y tzadeh et al. [138] presented a Multi-Objective Artificial Bee Colony (MOABC) algorithm to solve multiple objective problems. The method makes use of intelligent foraging by various bee types and utilizes the information supplied by an external archive to maintain the good solutions. A multi-objective artificial bee colony algorithm for optimization of power and heating system was proposed by A tashkari et al. [139]. Zou et al. [140] proposed multi-objective variant of $\mathrm{ABC}$ algorithm by making use of pareto concept and maintaining nondominated solutions in an external archive. The algorithm does not employ employed bees or scouts and all bees are considered as onlooker bees. A r s u a g a Rios, Vega-Rodriguez and Prieto-Castrillo [141] extended ABC algorithm to solve job scheduling problem with focus on two objectives namely cost requirements and optimizing time. Akbari et al. [142] modified the MOABC algorithm by using a grid to control diversity over the fixed-size archive. Special features of the modified algorithm such as effective trajectory adjusting and maintaining the diversity guide pave the way to solve the multi-objective problems in better way. A bedinia and Barazandeh [143] proposed IABC algorithm for application in distributed generation, network planning and evaluation of load demand. Yahya and Saka [144] combined MOABC algorithm with Levy flights walks by employed bees to solve a non-linear CSLP problem. The resulting hybrid algorithm improves the balance of search processes of employed bees and onlooker bees.

$\mathrm{Li}$ and Y in [145] developed a hybrid algorithm for estimation of parameters in chaotic systems. The algorithm introduced a hybrid bee operator to integrate the exploration ability of DE with exploitation capability of ABC. In [146] the authors modified the employed bee phase of $\mathrm{ABC}$ by using best solutions as well as random numbers in local and global neighbourhoods thus providing balanced exploration and exploitation. Shah et al. [147] presented a hybrid ABC by incorporating the global best solution in solution search equation of employed and onlooker bees phase. The scout bee phase was also modified by including the best-guided strategy. B ansal et al. [148] proposed three modifications in ABC: Self-adaptive step size 
in employed bee phase, updates in two components with modified step size in onlooker bee phase, limit parameter based on fitness of solutions in scout bee phase. Yazdani and Meybodi [149] modified the neighbour search process of employed and onlooker bees by use of roulette wheel selection and a random number of dimensions in choosing targets. A new scout mechanism was also provided for improved diversity and local optima avoidance. Liang and Lee [150] proposed a modified artificial bee colony algorithm by employing several strategies such as solution sharing, instant update strategy, cooperative strategy, and population management. $\mathrm{Huang}$, Wang and Yang [151] introduced a new search equation based on the combination of elite solution pool strategy and block perturbation strategy for employed bees and another search equation based on the best solution of current swarm for onlooker bees. Some authors in [152] performed experiments on benchmark functions using different selection schemes of $\mathrm{ABC}$ and found encouraging results. An improved artificial bee colony algorithm for constrained optimization problems is proposed in [153]. The algorithm involves the use of rank selection mechanism for exploitation of food sources by onlooker bees. The algorithm proposes a new search mechanism by use of best-so-far solution, in addition to use of periodic boundary handling mode to maintain the population diversity.

\section{ABC applications in data clustering}

The $\mathrm{ABC}$ algorithm to solve clustering problems was developed by $\mathrm{Zhang}$, O u y a g and Ning [154] that adopted Deb's method instead of greedy selection process to tackle infeasible solutions. The method involves use of a tournament selection operator to compare two solutions in accordance with three heuristic rules [155]. The algorithm adopts the Forgy algorithm to assign data points to the clusters and updating of cluster centers. In this process, cluster centers are updated when all data points are assigned to the closest cluster centers [156]. Karaboga and Ozturk [157] applied ABC in data clustering for classification purpose and compared its performance with PSO and other algorithms. The algorithm produced successful clustering results in comparison to other techniques. Z o u et al. [158] modified the $\mathrm{ABC}$ by employing the cooperative strategy to find best solution with contribution of every individual. The final solutions are obtained in accordance with information from all individuals. $\mathrm{Zh}$ ang et al. [159] proposed Chaotic $\mathrm{ABC}$, a modified version of $\mathrm{ABC}$ to solve partitional clustering problems. In the algorithm, Rossler chaotic number generator was incorporated during initialization of parameters to ensure more robustness and better quality of solutions.

S a e edi, S a madzadegan and El-Sheimy [160] successfully applied $\mathrm{ABC}$ in finding optimal clusters during object extraction from multi dimensional LIght Detection And Ranging (LIDAR) data that is used in 3D modelling of urban areas. Abdulsalam and Bakar [161] modified ABC to detect cluster-based deviations that constitute important application domains. The process is completed in two stages and deviations are detected in terms of outlier factor values for each object. Banharnsakun, Sirinaovakul and Achalakul [162] added the 
concept of multiple patrilines in best-so-far $\mathrm{ABC}$ algorithm to improve the quality of solutions as well as computation speed. The patrilines introduced the distributed approach idea in the search space and hence the parallel execution across multiple units for effective exploration. $\mathrm{Ju}$ and $\mathrm{Xu}$ [163] applied ABC in k-Means clustering to overcome the local optimal problems and produce effective clusters. Finally, a recommendation list for the target users is generated using modified collaborative recommendation approach. Lei, Huang and $\mathrm{Zhang}$ [164] presented a modified version of $\mathrm{ABC}$ for data clustering problem. Inspired by the strategy of PSO, they introduced additional parameters in ABC for effective search process and enhanced accuracy. The algorithm was found efficient in clustering analysis of data about DNA microarray gene expression and data sets on ProteinProtein Interaction (PPI). W u, L e i and T i a n [165] applied ABC in proposing a new clustering method for PPI networks. The ABC helps in optimizing the search process of original functional flow model and obtains better clustering results.

Marinakis, Marinaki and Matsatsinis [166] developed a twophase Discrete ABC-GRASP algorithm that performs the task of feature selection in first phase using discrete $\mathrm{ABC}$. In the second phase GRASP, an iterative search algorithm is utilized to solve the clustering problem.

Karaboga and Ozturk [167] applied ABC in fuzzy clustering. Various tests performed on medical data proved the successful performance of $\mathrm{ABC}$ algorithm. Le i, Tian and $\mathrm{Wu}$ [168] proposed a new clustering model for PPI networks by use of $\mathrm{ABC}$ search mechanism to find the cluster centers and then performing clustering through Intuitionistic Fuzzy Clustering (IFC) method. $\mathrm{S} \mathrm{u}$ et al. [169] introduced some modifications in $\mathrm{ABC}$ such as variable length strings, mutation operations and scheme for candidate solutions to generate Variable string length $\mathrm{ABC}(\mathrm{VABC})$ algorithm. The proposed algorithm in combination with Fuzzy $\mathrm{C}$-Means was used to find fuzzy partitions with accuracy and proper convergence. $\mathrm{Y}$ an to et al. [170] modified the $\mathrm{ABC}$ algorithm to find the objective function of Fuzzy K-Partition objective function. The hybrid algorithm was implemented on categorical data sets and obtained better dunn index values.

Dilmac and Korurek [171] proposed a classifier called MABCC based on modified $\mathrm{ABC}$ and applied in ECG signal analysis. The classifier generated satisfactory results when compared with GA and PSO based classifiers. H s i e h and Ye h [172] developed a Grid Scheme-Least Squares Support Vector Machine (GS-LSSVM) learning paradigm for classification problems by integrating grid scheme into least squares support vector machine. The $\mathrm{ABC}$ algorithm is employed to optimize parameters and improve classification. S hukran et al. [173] modified $\mathrm{ABC}$ for use in classification rule mining having six components. The algorithm proved its performance during testing on UCI datasets and comparison with other classification algorithms. Schiezaro and Pedrini [174] applied ABC in UCI data sets for feature selection and proved superior classification accuracy in selected features for majority of the data sets.

Krishnamoorthi and Natarajan [175] proposed two modifications on $\mathrm{ABC}$ for clustering problems. In the first algorithm, they introduced k-mean operator in scout bee phase for better performance; in the second algorithm, FCM 
operator generates new solutions in scout bee phase based on employed bee and onlooker bee phases. Le e, Che ng and Jiang [176] developed an early-warning model for business prediction and successfully applied $\mathrm{ABC}$ based clustering in the prediction of business failure. The tests performed on data of listed companies prove the accuracy of proposed model. R a k s h it et al. [177] proposed ABC based clustering technique in improving the accuracy by reducing the redundancy in feature set. The results prove that $\mathrm{ABC}$ helps to enhance the accuracy by reducing the number of redundant features in the data set. Bharti and Singh [178] improved the exploitation ability by embedding chaotic local search and gradient search methods in $\mathrm{ABC}$. The solution search equation was also improved in employed and onlooker phase to generate global best solution. The modified algorithm generated better quality of solutions and enhanced convergence speed on three text datasets.

S ridhar et al. [179] integrated the concept of rule-based system in ABC to develop an online application for diagnosis in chilli plants. The two-stage expert system produced better and efficient solutions. Shanthi and Amalraj [180] proposed a hybrid approach with combination of $\mathrm{ABC}$ and Harmonic Search algorithms. The modified C-ABC algorithm is able to find the local optimal solutions and has been used to develop a new clustering method for neural networks. The tests proved its performance in terms of better convergence, enhanced exploration and avoiding local optima traps. Y a n et al. [181] developed a new version of $\mathrm{ABC}$ called Hybrid $\mathrm{ABC}$ algorithm for clustering by adding the crossover operator of GA to enhance the information exchange between bees and the searching ability of $\mathrm{ABC}$. The results prove the better convergence speed and accuracy as compared to other algorithms. Uzer, Yilmaz and In an [182] applied $\mathrm{ABC}$ for reducing the feature vector dimensions in a hybrid method and then applying support vector machine classifier on the selected data. The approach was successfully applied in diagnosis of liver and diabetes. Tan et al. [183] modified $\mathrm{ABC}$ by introducing a key initialization method to generate the initial cluster centers by the reference of former ones. The modified algorithm is able to enhance the quality of clustering in addition to reducing the latency. J i et al. [184] introduced ABC-K-Modes clustering algorithm for categorical data by use of traditional k-modes method. One-step k-modes procedure was used for solution search in employed and onlooker phase. The multi-source search technique was adopted for scout bees to accelerate the convergence of algorithm. Chaurasia and S ing h [185] presented a hybrid artificial bee colony algorithm to solve the registration area-planning problem. The algorithm manages to preserve the grouping property of the problem while generating new solutions. Ve $\mathrm{nk}$ ate $\mathrm{sh}$ and Singh [186] proposed two $\mathrm{ABC}$ versions for the multiple traveling salesperson problem with a single depot. The problem was evaluated using two different objective functions i.e. minimizing total distance travelled by all the salespersons and minimizing the maximum distance travelled by any salesperson. S und ar and Singh [187] developed a grouping based ABC algorithm to solve Blockmodel Problem. The proposed algorithm is able to preserve the grouping information as far as possible. 
Reisi, Moradi and Abdollahpouri [188] proposed a feature weighting based $\mathrm{ABC}$ algorithm for data clustering. The modified method involves the use of different weights to each feature during initialization phase to produce good clustering results. Alshamiri, Singh and Surampudi [189] hybridized the Extreme Learning Machine (ELM) approach in ABC algorithm in order to improve the quality of clustering. The ELM is used to increase the separability of the input data into a high-dimensional ELM feature space by way of nonlinear transformation and $\mathrm{ABC}$ is then applied to perform clustering within this feature space. To solve the clustering problems more effectively and efficiently, improvements in three phases of original ABC were reported in [190]. The proposed algorithm produced better results by incorporating K-Means algorithm in initialization phase, an improved search equation in onlooker bees phase, Hooke and Jeeves-based method in scout bees phase. The authors in [191] developed a hybrid $\mathrm{ABC}$ algorithm to enhance the exploitation as well as convergence speed of original $\mathrm{ABC}$. The proposed algorithm employs a modified initialization phase in order to generate better initial solutions. The onlooker bees phase is also modified by incorporating the variable tournament selection in place of mechanism of roulette wheel selection. The hybrid algorithm performs better on various indices and provides optimal clustering solutions on standard data sets.

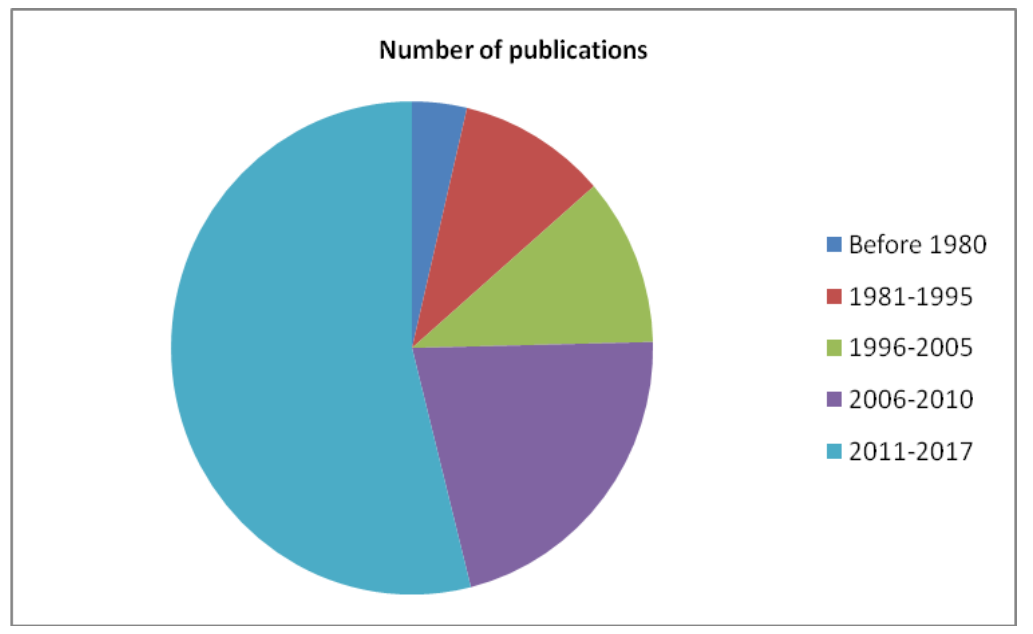

Fig. 1. Distribution of publications in the survey

\section{Conclusion}

This paper presents a review of previous research related to artificial bee colony algorithm, $\mathrm{ABC}$ variants and applications in data clustering. $\mathrm{ABC}$ is a simple and flexible algorithm and requires less parameters to be tuned in comparison to other meta-heuristic algorithms. It has become clear that original $\mathrm{ABC}$, its modifications, as well as hybrid algorithms are capable to solve a wide range of optimization problems including continuous, combinatorial, constrained, binary, multi-objective, 
and chaotic, etc. Various experiments performed in the relevant literature prove the efficiency, accuracy and effectiveness of $\mathrm{ABC}$ in solving several optimization problems.

$\mathrm{ABC}$ algorithms have been successfully implemented for data clustering problems and gave wonderful results leading to better prediction and analysis of data. In total, 191 papers have been reviewed, in which the concepts of swarm intelligence techniques are mentioned, and particular $\mathrm{ABC}$. It may be seen that the number of papers related to applications of $\mathrm{ABC}$ are increasing year by year. We hope that this survey will be very helpful for the researchers who are working in the area of $\mathrm{ABC}$ and data clustering. As seen from the studies given in the paper, more research is still required on $\mathrm{ABC}$ to overcome its limitations; development of new strategies is still required for generation and distribution of good solutions, faster convergence and improved exploitation for making $\mathrm{ABC}$ ideal in solving complex real world problems.

\section{References}

1. Wh e e l e r, W. M. The Ant-Colony as an Organism. - Journal of Morphology, Vol. 22, 1911, No 2, pp. 307-325.

2. Sulis, W. Fundamental Concepts of Collective Intelligence. - Nonlinear Dynamics, Psychology, and Life Sciences, Vol. 1, 1997, No 1, pp. 35-53.

3. B e n i, G., U. W a n g. Swarm Intelligence in Cellular Robotic Systems. - In: Proc. of NATO Advanced Workshop on Robots and Biological Systems, Tuscany, Italy, 1989.

4. D e n e u b o u r g, J. L., S. G o s s. Collective Patterns and Decision-Making. - Ethology Ecology \& Evolution, Vol. 1, 1989, pp. 295-311.

5. Theraulaz, G., J. L. Deneubourg. Swarm Intelligence in Social Insects and the Emergence of Cultural Swarm Patterns. Report No 92-09-046, Santa Fe Institute, Santa Fe, 1992.

6. Bonabeau, E., M. Dorigo, G. Theraulaz. Swarm Intelligence: From Natural to Artificial Systems. New York, Oxford University Press, Inc., USA, 1999.

7. Hin chey, M. G., R. S territt, C. R ouff. Swarms and Swarm Intelligence. - Computer, Vol. 40, 2007, pp. 111-113.

8. Kraus e, J., G. D. Ruxto n, S. Kraus e. Swarm Intelligence in Animals and Humans. Trends in Ecology and Evolution, Vol. 25, 2010, No 1, pp. 28-34.

9. Dorig o, M. Optimization, Learning and Natural Algorithm. Ph.D. Thesis, Politecnico di Milano, Italy, 1992.

10. K e n n e d y, J., R. E b e r h a r t. Particle Swarm Optimization. - In: Proc. of IEEE International Conference on Neural Networks IV, 1995, pp. 1942-1948.

11. Timmis, J., M. Neal, J. Hunt. An Artificial Immune System for Data Analysis. BioSystems, Vol. 55, 2000, pp. 143-150.

12. P a s s i n o, K. M. Biomimicry of Bacterial Foraging for Distributed Optimization and Control. IEEE Control Systems Magazine, Vol. 22, 2002, pp. 52-67.

13. Karaboga, D. An Idea Based on Honey Bee Swarm for Numerical Optimization. In: Technical Report - TR06, Erciyes University, 2005.

14. Chu, S. C., P. W. Tsai. Computational Intelligence Based on the Behavior of Cats. International Journal of Innovative Computing, Information and Control, Vol. 3, 2007, No 1, pp. 163-173.

15. Y a n g, X. S., S. D e b. Cuckoo Search via Levy Flights. - In: Proc. of the World Congress on Nature \& Biologically Inspired Computing (NaBIC'2009), Coimbatore, 2009, pp. 210-214.

16. Y a n g, X. S. Firefly Algorithms for Multimodal Optimization. - In: Stochastic Algorithms: Foundations and Applications, Springer Berlin, Heidelberg, 2009, pp. 169-178. 
17. R a shedi, E., H. Nezamabadi-Pour, S. S aryazdi. GSA: A Gravitational Search Algorithm. - Information Sciences, Vol. 179, 2009, No 13, pp. 2232-2248.

18. G a n, G., C. M a, J. W u. Data Clustering: Theory, Algorithms, and Applications. ASA-SIAM Series on Statistics and Applied Probability, SIAM, Philadelphia, VA, 2007, ISBN: 9780898716238.

19. T a n, P. N., M. S t e i $\mathrm{n} \mathrm{b}$ a c h, V. K u m a r. Introduction to Data Mining. Pearson Education, New Delhi, 3rd Edition, 2009.

20. S ingh, R. V., M. P. S. B h at i a. Data Clustering with Modified k-Means Algorithm. In: IEEE International Conference on Recent Trends in Information Technology (ICRTIT), Chennai, 2011, pp. 717-721.

21. X u, R., D. W u n s ch II. Survey of Clustering Algorithms. - IEEE Transactions on Neural Networks, Vol. 16, 2005, No 3, pp. 645-678.

22. J a i n, A. K., M. N. Murt y, P. J. F1 y n n. Data Clustering: A Review. - ACM Computing Surveys, Vol. 31, 1999, No 3, pp. 264-323.

23. H a n, J., M. K a mber. Data Mining: Concepts and Techniques. Second Edition. Morgan Kaufmann Publishers, California, USA, 2006.

24. K u m a r, Y., G. S a h o o. A Charged System Search Approach for Data Clustering. - Progress in Artificial Intelligence, Vol. 2, 2014, No 2, pp. 153-166.

25. D a y, W. H. E., H. Edels brunner. Efficient Algorithms for Agglomerative Hierarchical Clustering Methods. - Journal of Classification, Vol. 1, 1984, pp. 7-24.

26. M i c h a u d, P. Clustering Techniques. - Future Generation Computer Systems, Vol. 13, 1997, pp. 135-147.

27. J a i n, A. K., R. C. D u b e s. Algorithms for Clustering Data. Prentice-Hall, Inc., USA, 1988.

28. B e r k h i n, P. A Survey of Clustering Data Mining Techniques. - Grouping Multidimensional Data, 2006, pp. 25-71.

29. K a u f ma n, L., P. J. R o u s se e u w. Finding Groups in Data: An Introduction to Cluster Analysis. John Wiley and Sons, Inc., USA, 1990.

30. F i s h e r, W. D. On Grouping for Maximum Homogenity. - Journal of the American Statistical Association, Vol. 53, 1958, No 284, pp. 789-798.

31. Forgy, E. W. Cluster Analysis of Multivariate Data: Efficiency Versus Interpretability of Classification. - Biometrics, Vol. 21, 1965, pp. 768-769.

32. M a c q u e e n, J. Some Methods for Classification and Analysis of Multivariate Observations. In: L. Lecam, J. Neyman, Eds., Proc. of the 5th Berkeley Symposium on Mathematical Statistics and Probability, Theory of Statistics, University of California Press, USA, Vol. 1, 1967, pp. 281-297.

33. Nikn a m, T., E. T. F a rd, N. P o u r j a fari a n, A. R o u s t a. An Efficient Hybrid Algorithm Based on Modified Imperialist Competitive Algorithm and k-Means for Data Clustering. Engineering Applications of Artificial Intelligence, Vol. 24, 2011, pp. 306-317.

34. Krishna, K., M. Murty. Genetic k-Means Algorithm. - IEEE Transactions of Systems, Man, and Cybernetics, Part B: Cybernetics, Vol. 29, 1999, No 3, pp. 433-439.

35. Garai, G., B. B. Chaudhuri. A Novel Genetic Algorithm for Automatic Clustering. Pattern Recognition Letters, Vol. 25, 2004, pp. 173-187.

36. Maulik, U., S. B and yo padhyay. Genetic Algorithm-Based Clustering Technique. Pattern Recognition, Vol. 33, 2000, pp. 1455-1465.

37. La s z lo, M., S. M u kh e r je e. A Genetic Algorithm that Exchanges Neighboring Centers for k-Means Clustering. - Pattern Recognition Letters, Vol. 28, 2007, pp. 2359-2366.

38. S e 1 i m, S. Z., K. A $1 \mathrm{~s} \mathrm{u} 1 \mathrm{t}$ a n. A Simulated Annealing Algorithm for the Clustering Problem. Pattern Recognition, Vol. 24, 1991, No 10, pp. 1003-1008.

39. A l-S u $1 \mathrm{t}$ a n, K. S. A Tabu Search Approach to the Clustering Problem. - Pattern Recognition, Vol. 28, 1995, No 9, pp. 1443-1451.

40. S u n g, C. S., H. W. J i n. A Tabu-Search-Based Heuristic for Clustering. - Pattern Recognition, Vol. 33, 2000, pp. 849-858.

41. N g, M. K., J. C. W o n g. Clustering Categorical Data Sets Using Tabu Search Techniques. Pattern Recognition, Vol. 35, 2002, pp. 2783-2790.

42. K h a n, S. S., A. A h m a d. Cluster Center Initialization Algorithm for k-Means Clustering. Pattern Recognition Letters, Vol. 25, 2004, pp. 1293-1302. 
43. Redmond, S. J., C. Heneghan. A Method for Initializing the k-Means Clustering Algorithm Using kd-Trees. - Pattern Recognition Letters, Vol. 28, 2007, pp. 965-973.

44. Zalik, K. R. An Efficient k-Means Clustering Algorithm. - Pattern Recognition Letters, Vol. 29, 2008, pp. 1385-1391.

45. Shelokar, P. S., V. K. Jay araman, B. D. Kulkarni. An Ant Colony Approach for Clustering. - Analytica Chimica Acta, Vol. 509, 2004, pp. 187-195.

46. M e rw e, D. W., A. P. E n g e l b r e c h t. Data Clustering Using Particle Swarm Optimization. In: IEEE Congress on Evolutionary Computation (CEC'03), Canberra, 2003, pp. 215-220.

47. Cohen, S. C. M., L. N. de Castro. Data Clustering with Particle Swarms. - In: IEEE Congress on Evolutionary Computations, Vancouver, 2006, pp. 1792-1798.

48. A 1 a m, S., G. D o b b i e, P. R i d d l e. An Evolutionary Particle Swarm Optimization Algorithm for Data Clustering. - In: IEEE Swarm Intelligence Symposium, USA, 2008.

49. K a o, Y. T., E. Z a h a r a, I. W. K a o. A Hybridized Approach to Data Clustering. - Expert Systems with Applications, Vol. 34, 2008, pp. 1754-1762.

50. Y an g, F., T. Su n, C. Zhang. An Efficient Hybrid Data Clustering Method Based on k-Harmonic Means and Particle Swarm Optimization. - Expert Systems with Applications, Vol. 36, 2009, pp. 9847-9852.

51. B l u m, C., A. R o li. Metaheuristics in Combinatorial Optimization: Overview and Conceptual Comparison. - ACM Computing Surveys, Vol. 35, 2003, No 3, pp. 268-308.

52. B i a chi, L., M. Dorigo, L. M. Gambardella, W. J. Gutjahr. A Survey on Metaheuristics for Stochastic Combinatorial Optimization. - Natural Computing, Vol. 8, 2009, pp. 239-287.

53. N i k n a m, T., B. A m i ri. An Efficient Hybrid Approach Based on PSO, ACO and k-Means for Cluster Analysis. - Applied Soft Computing, Vol. 10, 2010, pp. 183-197.

54. Karaboga, D., B. Akay, C. Ozturk. Artificial Bee Colony (ABC) Optimization Algorithm for Training Feed-Forward Neural Networks. - In: Modeling Decisions for Artificial Intelligence, LNCS, Vol. 4617, Springer-Verlag, 2007, pp. 318-329.

55. K a r a b o g a, N. A New Design Method Based on Artificial Bee Colony Algorithm for Digital IIR Filters. - Journal of the Fraklin Institute, Vol. 346, 2009, pp. 328-348.

56. Okdem, S., D. Karaboga, C. Ozturk. An Application of Wireless Sensor Network Routing Based on Artificial Bee Colony Algorithm. - In: IEEE Congress on Evolutionary Computation (CEC), 2011, pp. 326-330.

57. R a o, R. V., P. J. P a w a r. Modelling and Optimization of Process Parameters of Wire Electrical Discharge Machining. - In: Proc. of the Institution of Mechanical Engineers, Part B: Journal of Engineering Manufacture, Vol. 223, 2009, No 11, pp. 1431-1440.

58. Lucic, P., D. Te odorovic. Computing with Bees: Attacking Complex Transportation Engineering Problems. - International Journal on Artificial Intelligence Tools, Vol. 12, 2003, No 3, pp. 375-394.

59. Te odorovic, D., M. Dell'Orco. Bee Colony Optimization - A Cooperative Learning Approach to Complex Transportation Problems. - In: Proc. of 10th EWGT Meeting, Poznan, 2005.

60. Te od orovic, D., P. L u c i c, G. M ark ovi c, M. De 1l'O r c o. Bee Colony Optimization: Principles and Applications. - In: 8th Seminar on Neural Network Applications in Electrical Engineering, NEUREL'06, Belgrade, 2006, pp. 151-156.

61. K a r a b o ga, D., B. Gorke m li, C. O z t u rk, N. K a r a b o ga. A Comprehensive Survey: Artificial Bee Colony (ABC) Algorithm and Applications. - Artificial Intelligence Review, Vol. 42, 2014, No 1, pp. 21-57.

62. A b u-M o u t i, F. S., M. E. E 1-H a w a r y. Overview of Artificial Bee Colony (ABC) Algorithm and Its Applications. - In: IEEE International Systems Conference (SysCon), Vancouver, 2012, pp. 1-6.

63. B a lasubramani, K., K. Marcus. A Comprehensive Review of Artificial Bee Colony Algorithm. - International Journal of Computers and Technology, Vol. 5, 2013, No 1, pp. 15-28.

64. K u ma r, B., D. Ku ma r. A Review on Artificial Bee Colony Algorithm. - International Journal of Engineering and Technology, Vol. 2, 2013, No 3, pp. 175-186. 
65. C a m a z i n e, S., J. S n e y d. A Model of Collective Nectar Source Selection by Honey Bees: Self-Organization Through Simple Rules. - Journal of Theoretical Biology, Vol. 149, 1991, pp. 547-571.

66. S e e ley, T. D. Social Foraging by Honeybees: How Colonies Allocate Foragers Among Patches of Flowers. - Behav. Ecol. Sociobiol., Vol. 19, 1986, pp. 343-354.

67. Towne, W. F., J. L. Gould. The Spatial Precision of the Honey Bees' Dance Communication. - Journal of Insect Behavior, Vol. 1, 1988, No 2, pp. 129-155.

68. R i b b a n d s, C. R. Division of Labour in the Honeybee Community. - In: Proc. R. Soc. Lond. B, Vol. 140, 1952, pp. 32-43.

69. All e n, M. D. The Honeybee Queen and Her Attendants. - Animal Behaviour, Vol. 8, 1960, pp. 201-208.

70. B e c k e r s, R., J. L. D e n e u b o u r g, S. G o s s, J. M. P a s t e e 1 s. Collective Decision Making through Food Recruitment. - Insectes Sociaux, Vol. 37, 1990, pp. 258-267.

71. S e e le y, T., S. C a m a z i n e, J. S n e y d. Collective Decision-Making in Honey Bees: How Colonies Choose Among Nectar Sources. - Behav. Ecol. Sociobiol., Vol. 28, 1991, pp. 277-290.

72. C a m a zin e, S. Self-Organizing Pattern Formation on the Combs of Honey Bee Colonies. Behav. Ecol. Sociobiol., Vol. 28, 1991, pp. 61-76.

73. H e i n r i c h, B. The Mechanisms and Energetics of Honeybee Swarm Temperature Regulation. - Journal of Experimental Biology, Vol. 91, 1981, pp. 25-55.

74. B on abeau, E., G. Theraulaz, J. L. Den eubourg, S. Aron, S. C a mazine. SelfOrganization in Social Insects. - Trends in Ecol. Evol., Vol. 12, 1997, pp. 188-193.

75. B on abeau, E., A. Sobkowski, G. Theraulaz, J. L. Den e ubourg. Adaptive Task Allocation Inspired by a Model of Division of Labor in Social Insects. - In: Proc. of BioComputing and Emergent Computation BCEC'97, World Scientific Press, 1997, pp. 36-45.

76. R o b i n s o n, G. E. Regulation of Division of Labor in Insect Societies. - Annu. Rev. Entomol., Vol. 37, 1992, pp. 637-665.

77. B a sturk, B., D. Karaboga. An Artificial Bee Colony (ABC) Algorithm for Numeric Function Optimization. - In: IEEE Swarm Intelligence Symposium 2006, Indiana, USA, 2006.

78. K a r a b o ga, D., B. B a s t u r k. A Powerful and Efficient Algorithm for Numerical Function Optimization: Artificial Bee Colony (ABC) Algorithm. - J. Glob. Optim., Vol. 39, 2007, pp. 459-471.

79. Karaboga, D., B. B a sturk. Artificial Bee Colony (ABC) Optimization Algorithm for Solving Constrained Optimization Problems. LNCS: Advances in Soft Computing Foundation of Fuzzy Logic and Soft Computing, LNCS 4529, Springer-Verlag, 2007, pp. 789-798.

80. Karaboga, D., B. Akay, C. Ozturk. Artificial Bee Colony (ABC) Optimization Algorithm for Training Feed-Forward Neural Networks. - In: V. Torra, Y. Narukawa, Y. Yoshida, Eds., MDAI 2007, LNAI 4617, Berlin, Heidelberg, Springer, 2007, pp. 318-329.

81. Karaboga, D., B. B a sturk. On the Performance of Artificial Bee Colony (ABC) Algorithm. - Applied Soft Computing, Vol. 8, 2008, pp. 687-697.

82. K a r a boga, D., B. Aka y. A Comparative Study of Artificial Bee Colony Algorithm. Applied Mathematics and Computation, Vol. 214, 2009, pp. 108-132.

83. Li u, H., L. G a o, X. Kong, S. Zh en g. An Improved Artificial Bee Colony Algorithm. In: 25th Chinese Control and Decision Conference (CCDC), Guiyang, China, 2013, pp. 401-404.

84. Z h u, G., S. K w o n g. Gbest-Guided Artificial Bee Colony Algorithm for Numerical Function Optimization. - Applied Mathematics and Computation, Vol. 217, 2010, pp. 3166-3173.

85. J a d o n, S. S., J. C. B a n s a l, R. Tiw a ri, H. S h a r m a. Expedited Artificial Bee Colony Algorithm. - In: M. Pant et al., Eds., Proc. of the Third International Conference on Soft Computing for Problem Solving, Advances in Intelligent Systems and Computing, Vol. 259, 2014, pp. 787-800.

86. E 1-A b d, M. Local Best Artificial Bee Colony Algorithm with Dynamic Sub-Populations. In: 2013 IEEE Congress on Evolutionary Computation, Cancun, Mexico, 2013, pp. 522-528. 
87. Fister, I., I. J r. Fis te r, J. B rest, V. Zu mer. Memetic Artificial Bee Colony Algorithm for Large-Scale Global Optimization. - In: 2012 IEEE World Congress on Computational Intelligence (WCCI), Brisbane, Australia, 2012.

88. B ansal, J. C., H. Sharma, K. V. Arya, A. Nagar. Memetic Search in Artificial Bee Colony Algorithm. - Soft Computing, Vol. 17, 2013, No 10, pp. 1911-1928.

89. Ku m a r, S., V. K. S h a r m a, R. Ku mari. Randomized Memetic Artificial Bee Colony Algorithm. - International Journal of Emerging Trends and Technology in Computer Science (IJETTCS), Vol. 3, 2014, No 1, pp. 52-62.

90. K o j i m a, M., H. N a k a n o, A. Mi y a u c hi. An Artificial Bee Colony Algorithm for Solving Dynamic Optimization Problems. - In: 2013 IEEE Congress on Evolutionary Computation, Cancun, 2013, pp. 2398-2405.

91. Y u, W., J. Z h a n g, W. Che n. Adaptive Artificial Bee Colony Optimization. - In: Proc. of 15th Annual Conference on Genetic and Evolutionary Computation (GECCO'13), Amsterdam, 2013, pp. 153-158.

92. Brajevic, I., M. Tuba. An Upgraded Artificial Bee Colony (ABC) Algorithm for Constrained Optimization Problems. - J. Intell. Manuf., Vol. 24, 2013, pp. 729-740.

93. Karaboga, D., B. Akay. A Modified Artificial Bee Colony (ABC) Algorithm for Constrained Optimization Problems. - Applied Soft Computing, Vol. 11, 2011, pp. 3021-3031.

94. Li, X., M. Y i n. Self-Adaptive Constrained Artificial Bee Colony for Constrained Numerical Optimization. - Neural Computing and Applications, Vol. 24, 2014, No 3, pp. 723-734.

95. A k a y, B., D. K a r a b o g a. Artificial Bee Colony Algorithm for Large Scale Problems and Engineering Design Optimization. - J. Intell. Manuf., Vol. 23, 2012, pp. 1001-1014.

96. K a s ha n, M. H., N. N a h a vandi, A. H. K a s h a n. DisABC: A New Artificial Bee Colony Algorithm for Binary Optimization. - Applied Soft Computing, Vol. 12, 2012, pp. 342-352.

97. P a m p a r a, G., A. P. E n g e 1 b r e c h t. Binary Artificial Bee Colony Optimization. - In: 2011 IEEE Symposium on Swarm Intelligence (SIS), Paris, 2011, pp. 1-8.

98. Chandras ekara n, K., S. He ma malin i, S. P. S i mon, N. P. P adh y. Thermal Unit Commitment Using Binary/Real Coded Artificial Bee Colony Algorithm. - Electric Power Systems Research, Vol. 84, 2012, pp. 109-119.

99. K i m, S. S., J. H. B y e o n, H. L i u, A. A b r a h a m, S. M c 1 o o n e. Optimal Job Scheduling in Grid Computing Using Efficient Binary Artificial Bee Colony Optimization. - Soft Computing, Vol. 17, 2013, pp. 867-882.

100. S in g h, A. An Artificial Bee Colony Algorithm for the Leaf-Constrained Minimum Spanning Tree Problem. - Applied Soft Computing, Vol. 9, 2009, pp. 625-631.

101. P a n, Q. K., M. F. T a s g e tire n, P. N. S u g a n th a n, T. J. Chu a. A Discrete Artificial Bee Colony Algorithm for the Lot-Streaming Flow Shop Scheduling Problem. - Information Sciences, Vol. 181, 2011, pp. 2455-2468.

102. Yurtkuran, A., E. Emel. A Modified Artificial Bee Colony Algorithm for P-Center Problems. - The Scientific World Journal, Article id 824196, 2014. 9 p.

103. Li, J. Q., Q. K. P a n, K. Z. G a o. Pareto-Based Discrete Artificial Bee Colony Algorithm for Multi-Objective Flexible Job Shop Scheduling Problems. - Int. J. Adv. Manuf. Technol., Vol. 55, 2011, pp. 1159-1169.

104. B e l o u f a, F., M. A. C h i k h. Design of Fuzzy Classifier for Diabetes Disease Using Modified Artificial Bee Colony Algorithm. - Computer Methods and Programs in Biomedicine, Vol. 112, 2013, No 1, pp. 92-103.

105. Khors andi, A., S. H. Hos seinian, A. Ghaz a n fari. Modified Artificial Bee Colony Algorithm Based on Fuzzy Multi-Objective Technique for Optimal Power Flow Problem. Electric Power Systems Research, Vol. 95, 2013, pp. 206-213.

106. Di w o ld, K., A. A d e rhold, A. S ch e id le r, M. Midd e n d o r f. Performance Evaluation of Artificial Bee Colony Optimization and New Selection Schemes. - Memetic Comp., Vol. 3, 2011, pp. 149-162.

107. A b r a ha m, A., R. K. J a to th, A. R a j a s e k har. Hybrid Differential Artificial Bee Colony Algorithm. - Journal of Computational and Theoretical Nanoscience, Vol. 9, 2012, pp. 1-9. 
108. A bro, A. G., J. Mohamad-S a leh. An Enhanced Artificial Bee Colony Optimization Algorithm. - In: D. S. Nikos Mastorakis, Valeriu Prepelita, Eds., WSEAS Press, Recent Advances in Systems Science and Mathematical Modeling, 2012, pp. 222-227.

109. Abro, A. G., J. Mohamad-S a leh. Enhanced Global-Best Artificial Bee Colony Optimization Algorithm. - In: Sixth UKSim/AMSS European Symposium on Computer Modeling and Simulation (EMS), Valetta, Malta, 2012, pp. 95-100.

110. Li, G., P. N i u, X. X i a o. Development and Investigation of Efficient Artificial Bee Colony Algorithm for Numerical Function Optimization. - Applied Soft Computing, Vol. 12, 2012, pp. 320-332.

111. A b r o, A. G., J. M o h a m a d-S a leh. Enhanced Probability-Selection Artificial Bee Colony Algorithm for Economic Load Dispatch: A Comprehensive Analysis. - Engineering Optimization, Vol. 46, 2014, No 10, pp. 1315-1330.

112. Sh arma, H., J. C. B ansa l, K. V. Arya. Opposition Based Levy Flight Artificial Bee Colony. - Memetic Computing, Vol. 5, 2013, No 3, pp. 213-227.

113. Xu, Y., P. Fan, L. Yu an. A Simple and Efficient Artificial Bee Colony Algorithm. Mathematical Problems in Engineering, Article ID 526315, 2013. 9 p.

114. Kan g, F., J. Li, H. Li. Artificial Bee Colony Algorithm and Pattern Search Hybridized for Global Optimization. - Applied Soft Computing, Vol. 13, 2013, pp. 1781-1791.

115. Tsa i, P. W., J. S. Pan, B. Y. Li a o, S. C. Chu. Enhanced Artificial Bee Colony Optimization. - International Journal of Innovative Computing, Information and Control, Vol. 5, 2009, No 12, pp. 1-12.

116. A l at a s, B. Chaotic Bee Colony Algorithms for Global Numerical Optimization. - Expert Systems with Applications, Vol. 37, 2010, 5682-5687.

117. K i r a n, M. S., M. G u n d u z. A Novel Artificial Bee Colony Based Algorithm for Solving the Numerical Optimization Problems. - International Journal of Innovative Computing, Information and Control, Vol. 8, 2012, No 9, pp. 6107-6121.

118. Dongli, Z., G. Xinping, T. Ying g n, T. Yong. Modified Artificial Bee Colony Algorithms for Numerical Optimization. - In: 3rd International Workshop on Intelligent Systems and Applications (ISA), Wuhan, China, 2011, pp. 1-4.

119. Dongli, Z., G. Xinping, T. Yinggan, T. Yong. An Artificial Bee Colony Optimization Algorithm Based on Multi-Exchange Neighborhood. - In: Fourth International Conference on Computational and Information Sciences (ICCIS), Chongqing, China, 2012, pp. 211-214.

120. B a n h arn s a ku n, A., T. A c h a la ku l, B. S i r in a ov a ku l. The Best-So-Far Selection in Artificial Bee Colony Algorithm. - Applied Soft Computing, Vol. 11, 2011, pp. 2888-2901.

121. Ga o, W., S. Li u. Improved Artificial Bee Colony Algorithm for Global Optimization. Information Processing Letters, Vol. 111, 2011, pp. 871-882.

122. Ga o, W., S. Li u, L. Hu ang. A Global Best Artificial Bee Colony Algorithm for Global Optimization. - Journal of Computational and Applied Mathematics, Vol. 236, 2012, pp. 2741-2753.

123. Ga o, W., S. Li u. A Modified Artificial Bee Colony Algorithm. - Computers \& Operations Research, Vol. 39, 2012, pp. 687-697.

124. Ga o, W. F., S. Y. Li u, L. L. Hu an g. A Novel Artificial Bee Colony Algorithm Based on Modified Search Equation and Orthogonal Learning. - IEEE Transactions on Cybernetics, Vol. 43, 2013, No 3, pp. 1011-1024.

125. Sharma, T. K., M. Pant. Enhancing the Food Locations in an Artificial Bee Colony Algorithm. - Soft Computing, Vol. 17, 2013, No 10, pp. 1939-1965.

126. X i a n g, W., M. A n. An Efficient and Robust Artificial Bee Colony Algorithm for Numerical Optimization. - Computers \& Operations Research, Vol. 40, 2013, pp. 1256-1265.

127. B a n sa l, J. C., H. S h arma, A. N a gar, K. V. Arya. Balanced Artificial Bee Colony Algorithm. - Int. J. Artificial Intelligence and Soft Computing, Vol. 3, 2013, No 3, pp. 222-243.

128. B i s was, S., S. Das, S. Debchoudhury, S. Kundu. Co-Evolving Bee Colonies by Forager Migration: A Multi-Swarm Based Artificial Bee Colony Algorithm for Global Search Space. - Applied Mathematics and Computation, Vol. 232, 2014, pp. 216-234. 
129. Lu o, J., Q. Wang, X. Xi a o. A Modified Artificial Bee Colony Algorithm Based on Converge-Onlookers Approach for Global Optimization. - Applied Mathematics and Computation, Vol. 219, 2013, pp. 10253-10262.

130. S u 1 a i m a n, N., J. M. S a le h, A. G. A b r o. A Modified Artificial Bee Colony (JA-ABC) Optimization Algorithm. - In: Proc. of International Conference on Applied Mathematics and Computational Methods in Engineering, 2013, pp. 74-79.

131. G a o, W. F., S. Y. Li u, L. L. H u a n g. A Novel Artificial Bee Colony Algorithm with Powell's Method. - Applied Soft Computing, Vol. 13, 2013, No 9, pp. 3763-3775.

132. D a s, K. N., B. C h a u d h u r. Modified Activity of Scout Bee in ABC for Global Optimization. - In: M. Pant et al., Eds., Proc. of 3rd International Conference on Soft Computing for Problem Solving, Advances in Intelligent Systems and Computing, Vol. 259, 2014, pp. 649-659.

133. A k a y, B., D. K a r a b o g a. A Modified Artificial Bee Colony Algorithm for Real-Parameter Optimization. - Information Sciences, Vol. 192, 2012, pp. 120-142.

134. A 1 i z a d e g a n, A., B. A s a d y, M. A h m a d p o u r. Two Modified Versions of Artificial Bee Colony Algorithm. - Applied Mathematics and Computation, Vol. 225, 2013, pp. 601-609.

135. Li an g, Y., Y. Li u, L. Zh an g. An Improved Artificial Bee Colony (ABC) Algorithm for Large Scale Optimization. - In: 2nd International Symposium on Instrumentation and Measurement, Sensor Network and Automation (IMSNA), Toronto, 2013, pp. 644-648.

136. A y di n, D., T. Li a o, M. A. Montes de O c a, T. S tutzl e. Improving Performance via Population Growth and Local Search: The Case of the Artificial Bee Colony Algorithm. In: J.-K. Hao et al., Eds., EA 2011, LNCS 7401, Berlin, Springer, 2012, pp. 85-96.

137. Omkar, S. N., J. Senthilnath, R. Khandelwal, G. N. Naik, S. Gopalakrishnan. Artificial Bee Colony (ABC) for Multi-Objective Design Optimization of Composite Structures. - Applied Soft Computing, Vol. 11, 2011, pp. 489-499.

138. He d a y t z a de h, R., B. Has a n i zadeh, R. A k b a ri, K. Zi a r at i. A Multi-Objective Artificial Bee Colony for Optimizing Multi-Objective Problems. - In: 3rd International Conference on Advanced Computer Theory and Engineering (ICACTE), Chengdu, 2010, pp. 271-281.

139. At a shkari, K., N. Narimanzadeh, A. R. Ghavimi, M. J. Mahmoodabadi, F. A g h a i e n e zh a d. Multi-Objective Optimization of Power and Heating System Based on Artificial Bee Colony. - In: International Symposium on Innovations in Intelligent Systems and Applications (INISTA), Istanbul, 2011, pp. 64-68.

140. Z ou, W., Y. Zhu, H. Ch en, H. S h e n. A Novel Multi-Objective Optimization Algorithm Based on Artificial Bee Colony. - In: Proc. of 13th Annual Conference Companion on Genetic and Evolutionary Computation, GECCO'11, Dublin, 2011, pp. 103-104.

141. Arsuaga-Rios, M., M. A. Vega-Rodriguez, F. Prieto-Castrillo. MultiObjective Artificial Bee Colony for Scheduling in Grid Environments. - In: IEEE Symposium on Swarm Intelligence (SIS), Paris, 2011, pp. 1-7.

142. A k b a ri, R., R. He d a y a $\mathrm{z}$ a d e h, K. Zi ar a ti, B. H a s s a n i z a d e h. A Multi-Objective Artificial Bee Colony Algorithm. - Swarm and Evolutionary Computation, Vol. 2, 2012, pp. 39-52.

143. A b e din i a, O., E. S. B a r a z a n d e h. Interactive Artificial Bee Colony Based on Distribution Planning with Renewable Energy Units. - In: IEEE PES Innovative Smart Grid Technologies (ISGT), Washington, 2013, pp. 1-6.

144. Y a h y a, M., M. P. S a k a. Construction Site Layout Planning Using Multi-Objective Artificial Bee Colony Algorithm with Levy Flights. - Automation in Construction, Vol. 38, 2014, pp. 14-29.

145. Li, X., M. Y i n. Parameter Estimation for Chaotic Systems by Hybrid Differential Evolution Algorithm and Artificial Bee Colony Algorithm. - Nonlinear Dynamics, Vol. 77, 2014, No 1, pp. 61-71.

146. J a d o n, S. S., J. C. B a n s a l, R. Ti w a r i, H. S h a r m a. Artificial Bee Colony Algorithm with Global and Local Neighborhoods. - International Journal of System Assurance Engineering and Management, 2014, pp. 1-13. 
147. S h a h, H., T. H e r a w a n, R. N a s e e m, R. G h a z a li. Hybrid Guided Artificial Bee Colony Algorithm for Numerical Function Optimization. - In: Y. Tan et al., Eds., ICSI 2014, Part I. LNCS 8794, Berlin, Springer, 2014, pp. 197-206.

148. B a n s a 1, J. C., H. S h a r m a, K. V. A r y a, K. D e e p, M. P a n t. Self-Adaptive Artificial Bee Colony. - Optimization, Vol. 63, 2014, No 10, pp. 1513-1532.

149. Y a zdani, D., M. R. Meybodi. A Novel Artificial Bee Colony Algorithm for Global Optimization. - In: Proc. of 4th International e-Conference on Computer and Knowledge Engineering (ICCKE), Mashhad, Iran, 2014, pp. 443-448.

150. Li a n g, J.-H., C.-H. L e e. A Modification Artificial Bee Colony Algorithm for Optimization Problems. - Mathematical Problems in Engineering, Vol. 2015, 2015, Article ID 581391. $13 \mathrm{p}$.

151. H u a n g, F., L. W a n g, C. Y a n g. A New Improved Artificial Bee Colony Algorithm for Ship Hull Form Optimization. - Engineering Optimization, Vol. 48, 2016, No 4, pp. 672-686.

152. K u m a r, A., D. K u m a r, S. K. J a r i a l. A Comparative Analysis of Selection Schemes in the Artificial Bee Colony Algorithm. - Computacion y Sistemas, Vol. 20, 2016, No 1, pp. 55-66.

153. Li a n g, Y., Z. W a n, D. F an g. An Improved Artificial Bee Colony Algorithm for Solving Constrained Optimization Problems. - International Journal of Machine Learning and Cybernetics, Vol. 8, 2017, No 3, pp. 739-754.

154. Zh a n g, C., D. Ou y an g, J. Ning. An Artificial Bee Colony Approach for Clustering. Expert Systems with Applications, Vol. 37, 2010, pp. 4761-4767.

155. G o l d b e r g, D. E., K. D e b. A Comparative Analysis of Selection Schemes Used in Genetic Algorithms. - In: GJE Rawlins, Eds., Foundations of Genetic Algorithms, 1991, pp. 69-93.

156. Forgy, E. W. Cluster Analysis of Multivariate Data: Efficiency Versus Interpretability of Classification. - Biometrics, Vol. 21, 1965, pp. 768-769.

157. Karaboga, D., C. Ozturk. A Novel Clustering Approach: Artificial Bee Colony (ABC) Algorithm. - Applied Soft Computing, Vol. 11, 2011, pp. 652-657.

158. Z ou, W., Y. Zhu, H. Che n, X. S u i. A Clustering Approach Using Cooperative Artificial Bee Colony Algorithm. - Discrete Dynamics in Nature and Society, Vol. 2010, Article id 459796, 2010. $16 \mathrm{p}$.

159. Zhang, Y., L. W u, S. W ang, Y. Hu o. Chaotic Artificial Bee Colony Used for Cluster Analysis. - In: R. Chen, Eds., Intelligent Computing and Information Science, Communications in Computer and Information Science, Springer-Berlin, Vol. 134, 2011, No 1, pp. 205-211.

160. S a e e d i, S., F. S a m a d z a d e g a n, N. E l-S h e i m y. Object Extraction from LIDAR Data Using an Artificial Swarm Bee Colony Clustering Algorithm. - In: U. Stilla, F. Rottensteiner, N. Paparoditis, Eds., CMRT'09, IAPRS, Vol. 38, 2009, pp. 133-138.

161. A bdu $1 \mathrm{~s}$ a la m, M. F., A. A. B a k a r. A Cluster-Based Deviation Detection Task Using the Artificial Bee Colony (ABC) Algorithm. - International Journal of Soft Computing, Vol. 7, 2012, No 2, pp. 71-78.

162. B a nharn sakun, A., B. S irin a ovakul, T. A chal a kul. The Best-So-Far ABC with Multiple Patrilines for Clustering Problems. - Neurocomputing, Vol. 116, 2013, pp. 355-366.

163. J u, C., C. X u. A New Collaborative Recommendation Approach Based on Users Clustering Using Artificial Bee Colony Algorithm. - The Scientific World Journal, Vol. 2013, Article id $869658,2013.9$ p.

164. Lei, X., X. Huang, A. Zhang. Improved Artificial Bee Colony Algorithm and Its Application in Data Clustering. - In: IEEE 5th International Conference on Bio-Inspired Computing: Theories and Applications (BIC-TA), Changsha, China, 2010, pp. 514-521.

165. W u, S., X. L e i, J. T i a n. Clustering PPI Network Based on Functional Flow Model through Artificial Bee Colony Algorithm. - In: 7th International Conference on Natural Computation (ICNC), Shanghai, 2011, pp. 92-96.

166. Marinakis, Y., M. Marinaki, N. Matsatsinis. A Hybrid Discrete Artificial Bee Colony - GRASP Algorithm for Clustering. - In: International Conference on Computers and Industrial Engineering (CIE'2009), Troyes, France, 2009, pp. 548-553.

167. Karaboga, D., C. Ozturk. Fuzzy Clustering with Artificial Bee Colony Algorithm. Scientific Research and Essays, Vol. 5, 2010, No 14, pp. 1899-1902. 
168. L e i, X., J. T i a n, F. W u. PPI Modules Detection Method Through ABC-IFC Algorithm. - In: IEEE International Conference on Bioinformatics and Biomedicine (BIBM), Shanghai, 2013.

169. S u, Z.-G., P.-H. W an g, J. S h e n, Y.-G. Li, Y.-F. Z h an g, E.-J. H u. Automatic Fuzzy Partitioning Approach Using Variable String Length Artificial Bee Colony (VABC) Algorithm. - Applied Soft Computing, Vol. 12, 2012, pp. 3421-3441.

170. Y a n to, I. T. R., Y. S a adi, D. Hart a ma, D. P. I s mi, A. Pranolo. A Framework of Fuzzy Partition Based on Artificial Bee Colony for Categorical Data Clustering. - 2nd International Conference on Science in Information Technology (ICSITech), Balikpapan, Indonesia, 2016, pp. 260-263.

171. D i $1 \mathrm{~m}$ a c, S., M. K o r u r e k. A New ECG Arrhythmia Clustering Method Based on Modified Artificial Bee Colony Algorithm, Comparison with GA and PSO Classifiers. - In: IEEE International Symposium on Innovations in Intelligent Systems and Applications (INISTA), Albena, 2013, pp. 1-5.

172. H s i e h, T. J., W. C. Y e h. Knowledge Discovery Employing Grid Scheme Least Squares Support Vector Machines Based on Orthogonal Design Bee Colony Algorithm. - IEEE Transactions on Systems, Man, and Cybernetics - Part B: Cybernetics, Vol. 41, 2011, No 5, pp. 1198-1212.

173. Shukran, M. A. M., Y. Y. Chung, W. C. Yeh, N. W ahid, A. M. A. Z a id i. Artificial Bee Colony Based Data Mining Algorithms for Classification Tasks. - Modern Applied Science, Vol. 5, 2011, No 4, pp. 217-231.

174. S chiezaro, M., H. Pedrini. Data Feature Selection Based on Artificial Bee Colony Algorithm. - EURASIP Journal on Image and Video Processing, Vol. 47, 2013, pp. 1-8.

175. Krishnamoorthi, M., A. M. Natarajan. A Comparative Analysis of Enhanced Artificial Bee Colony Algorithms for Data Clustering. - In: International Conference on Computer Communication and Informatics (ICCCI'13), Coimbatore, 2013.

176. Le e, T. E., J. H. C h e n g, L. L. J i a n g. A New Artificial Bee Colony Based Clustering Method and its Application to the Business Failure Prediction. - In: International Symposium on Computer, Consumer and Control (IS3C), Taichung, 2012, pp. 72-75.

177. Rakshit, P., S. B hat t a char y y a, A. Kon ar, A. K h a s nobish, D. N. Tibarewal a, R. J a $\mathrm{arth}$ a $\mathrm{n}$ a n. Artificial Bee Colony Based Feature Selection for Motor Imagery EEG Data. - In: J. C. Bansal, Eds., Proceedings of Seventh International Conference on BioInspired Computing: Theories and Applications (BIC-TA 2012), AISC, Springer Berlin, Vol. 202, 2012, pp. 127-138.

178. B h a r t i, K. K., P. K. S i n g h. Chaotic Gradient Artificial Bee Colony for Text Clustering. Soft Computing, Vol. 20, 2016, No 3, pp. 1113-1126.

179. S rid h a r, D. V. P. R., M. S P. B a b u, M. P a ri m a l a, N. T. R a o. Implementation of WebBased Chilli Expert Advisory System Using ABC Optimization Algorithm. - International Journal on Computer Science and Engineering, Vol. 2, 2010, No 6, pp. 2141-2144.

180. Shanthi, D., R. A malraj. Collaborative Artificial Bee Colony Optimization Clustering Using SPNN. - Procedia Engineering, Vol. 30, 2012, pp. 989-996.

181. Y a n, X., Y. Z h u, W. Z o u, L. W a n g. A New Approach for Data Clustering Using Hybrid Artificial Bee Colony Algorithm. - Neurocomputing, Vol. 97, 2012, pp. 241-250.

182. U z e r, M. S., N. Y i 1 m a z, O. I n a n. Feature Selection Method Based on Artificial Bee Colony Algorithm and Support Vector Machines for Medical Datasets Classification. - The Scientific World Journal, Vol. 2013, 2013, Article id 419187. 10 p.

183. T a n, Q., H. W u, B. Hu, X. X. Li u. An Improved Artificial Bee Colony Algorithm for Clustering. - In: Proc. of the Companion Publication of the 2014 Annual Conference on Genetic and Evolutionary Computation (GECCO Comp'14), Vancouver, 2014, pp. 19-20.

184. J i, J., W. P an g, Y. Z h en g, Z. W a n g, Z. M a. An Artificial Bee Colony Based Clustering Algorithm for Categorical Data. - PLoS ONE, Vol. 10, 2015, No 5, e0127125, doi: 10.1371/journal.pone.0127125.

185. Ch a u r a si a, S. C., A. S ing h. A Hybrid Swarm Intelligence Approach to the Registration Area Planning Problem. - Information Sciences, Vol. 302, 2015, pp. 50-69.

186. Venkatesh, P., A. S ingh. Two Metaheuristic Approaches for the Multiple Traveling Salesperson Problem. - Applied Soft Computing, Vol. 26, 2015, pp. 74-89. 
187. S und ar, S., A. Singh. Metaheuristic Approaches for the Blackmodel Problem. - IEEE Systems Journal, Vol. 9, 2015, No 4, pp. 1237-1247.

188. Reis i, M., P. Moradi, A. Abdollahpouri. A Feature Weighting Based Artificial Bee Colony Algorithm for Data Clustering. - In: Proc. of 8th International Conference on Information and Knowledge Technology (IKT), Hamedan, Iran, 2016, pp. 134-138.

189. A $1 \mathrm{~s} \mathrm{~h}$ a miri, A. K., A. S ingh, B. R. S u ra mpudi. Artificial Bee Colony Algorithm for Clustering: An Extreme Learning Approach. - Soft Computing, Vol. 20, 2016, No 8, pp. 3163-3176.

190. K u m a r, Y., G. S a h o o. A Two-Step Artificial Bee Colony Algorithm for Clustering. - Neural Computing and Applications, Vol. 28, 2017, No 3, pp. 537-551.

191. K u m a r, A., D. K u m a r, S. K. J a ri a l. A Novel Hybrid K-Means and Artificial Bee Colony Algorithm Approach for Data Clustering. - Decision Science Letters, Vol. 7, 2018, pp. 65-76. 